\title{
DIVERSIDAD Y RIQUEZA DE ESPECIES DE LA FAMILIA ARANEIDAE (ARACHNIDA, ARANEAE) EN CICRA (MADRE DE DIOS - PERÚ)
}

\section{DIVERSITY AND SPECIES RICHNESS OF THE FAMILY ARANEIDAE (ARACHNIDA, ARANEAE) IN CICRA (MADRE DE DIOS - PERU)}

\author{
Mariajosé Deza ${ }^{1}$ y Juan Manuel Andía ${ }^{2}$
}

\begin{abstract}
Resumen
El objetivo del presente estudio fue registrar la riqueza y diversidad de la familia Araneidae en CICRA. Se realizaron colectas cuantitativas en dos épocas marcadas: seca (ES: agosto setiembre, 2005) y lluviosa (ELL: febrero - marzo, 2006), eligiendo aleatoriamente 6 parcelas de 01 ha cada una, siendo las mismas en ambas temporadas. Se empleó la captura directa mediante colecta manual, utilizando los métodos Ground Hand Collecting (colecta hasta el nivel de la rodilla) y Aerial Hand Collecting (colecta desde el nivel de la rodilla hasta dos metros de altura aproximadamente). Cada método se realizó durante una hora, tanto en el día (7:00 AM - 9:00 AM) como en la noche (7:00 PM - 9:00 PM) obteniéndose un total de 24 muestras. En ES se colectaron 1516 individuos distribuidos en 25 géneros y 120 especies y en la ELL se colectaron 3173 individuos en 24 géneros y 121 especies, dando un total de 154 especies; de las cuales las más representativas en ambas épocas fueron Micrathena kirbyi (ES: 12\%, ELL: 22\%), Araneus sp. 1 (ES: 4.6\%, ELL: 5\%), Micrathena cf. exlinae (ES: 5.3\%, ELL: 3.7\%), Parawixia kochi (ES: 4\%, ELL: 6.3\%) y Cyclosa inca (ES: 6.7\%, ELL: 6\%). La diversidad obtenida en ES (H': 3.829) fue más alta que en ELL ( $\left.\mathrm{H}^{\prime}: 3.453\right)$ a pesar de que la ELL presentó una mayor riqueza y abundancia. Palabras clave: Araneae, Araneidae, diversidad, riqueza de especies, Madre de Dios - Perú
\end{abstract}

\begin{abstract}
The aim of the present study was to register richness and diversity of the family Araneidae in CICRA. Quantitative collections were made in two marked seasons: dry season (ES: august september, 2005) and rainy season (ELL: february - march, 2006), randomly choosing six plots of one hectare each, being the same for both seasons. Direct capture was used by means of manual collection, using the following methods: Ground Hand Collecting (collects up to the level of the knee) and Aerial Hand Collecting (collects from the level of the knee up to two meters of height approximately). Each method was applied during an hour, in the daytime (7:00 AM - 9:00 AM) and in the night (7:00 PM - 9:00 PM) obtaining 24 samples. In the dry season 1516 individuals were collected, distributed in 25 genera and 120 species; in the rainy season 3173 individuals were collected, distributed in 24 genera and 121 species, totaling $154 \mathrm{spp}$. The most representative species in both seasons were Micrathena kirbyi (ES: 12\%, ELL: 22\%), Araneus sp. 1 (ES: 4.6\%, ELL: 5\%), Micrathena cf. exlinae (ES: 5.3\%, ELL: 3.7\%), Parawixia kochi (ES: 4\%, ELL: 6.3\%) and Cyclosa inca (ES: 6.7\%, ELL: 6\%). The diversity obtained in ES (H': 3.829) was higher than in ELL ( $\left.\mathrm{H}^{\prime}: 3.453\right)$ in spite of the fact that the ELL presented major richness and abundance.
\end{abstract}

Key words: Araneae, Araneidae, diversity, species richness, Madre de Dios - Peru

\section{Introducción.}

Las arañas conforman uno de los grupos Megadiversos del planeta, con 40998 especies distribuidas en 3747 géneros (Platnick, 2009), de los cuales más de 1000 ocurren en el Neotrópico. Teniendo entera dependencia de la depredación como estrategia trófica, y por tanto, situándose en la parte más alta de la cadena alimenticia de los invertebrados, las arañas son un componente importante de cualquier ecosistema (Coddington et al., 1991) donde habiten. Dentro de este orden, la familia Araneidae, de distribución cosmopolita, se constituye como una de las más numerosas, con 2985 especies en el mundo (Platnick, 2009), con una estimación de 6000 a 7000 especies si se considera la alta diversidad y el alto grado de desconocimiento de los sistemas tropicales (Coddington \& Levi, 1991).

El Departamento de Madre de Dios es altamente diverso, por lo que más del $55 \%$ de su territorio se encuentra protegido en dos Parques Nacionales, una Reserva Comunal, una Reserva Nacional y una Concesión para la Conservación. Silva \& Coddington (1996) realizaron un estudio en este departamento, pero aún se está muy lejos de registrar la riqueza del lugar, ya que constantemente se están encontrando nuevas especies.

Este estudio tiene como finalidad registrar la riqueza y diversidad de la familia Araneidae en los 
terrenos de CICRA (Centro de Investigación y Capacitación río Los Amigos) que se encuentra en la Cuenca del río Los Amigos en Madre de Dios, en dos épocas marcadas, seca y lluviosa.

\section{Materiales y métodos.}

Área de estudio.

CICRA (Centro de Investigación y Capacitación río Los Amigos) se encuentra en la cuenca del río Los Amigos en el Departamento de Madre de Dios el cual es el tercero más grande de la Amazonía en el Perú, después de los departamentos de Loreto y Ucayali; el $82 \%$ está por debajo de los $500 \mathrm{~m}$ de elevación. La elevación de la terraza de CICRA es $41 \mathrm{~m}$ sobre el río Madre de Dios y aproximadamente $80 \mathrm{~m}$ encima de la ciudad de Puerto Maldonado. La elevación en CICRA es más alta que la mayor parte de la Amazonía norte del Perú. La concesión para la conservación en la que se encuentra CICRA tiene una extensión de 146051 ha, equivalente al $33 \%$ de la cuenca y tiene un perímetro de $450 \mathrm{Km}$. CICRA se ubica aproximadamente en los $12^{\circ} 34^{\prime} 07^{\prime \prime} \mathrm{S} 70^{\circ} 05^{\prime} 57^{\prime \prime} \mathrm{W}$ o en las coordenadas UTM 380500E $8610297 \mathrm{~N}$ 19S (Pitman, 2008).

Muestreos.

La recolección de datos se llevó a cabo en dos temporadas: 1) Temporada seca ( 23 de agosto al 2 de octubre 2005), registrándose una Temperatura promedio de $23.24^{\circ} \mathrm{C}\left(\mathrm{T}^{\mathrm{o}} \mathrm{min}\right.$. Prom. $12.91^{\circ} \mathrm{C}, \mathrm{T}^{\mathrm{o}}$ max. prom. $27.94^{\circ}$ ) y una humedad relativa promedio de $80.77 \%$ (HR min. Prom. 70.26\%, HR max. Prom. $93.53 \%$ ) y 2) Temporada lluviosa (14 de febrero al 19 marzo del 2006), registrándose una temperatura promedio de $25.92^{\circ} \mathrm{C}\left(\mathrm{T}^{\circ} \min\right.$. Prom. $20.23^{\circ} \mathrm{C}, \mathrm{T}^{\circ} \max$. prom. $27.63^{\circ} \mathrm{C}$ ) y una humedad relativa de $90.36 \%$ (HR min. Prom. 78.85\%, HR max. Prom. 99.37\%).

1. En campo.

- Se determinó aleatoriamente 6 parcelas de muestreo que se ubicaron cerca de las trochas Cocha Lobo (1), Darío (2), Otorongo (3), Segundo Mirador (4), Castañal (5) y Palmeras (6). Cada parcela de muestreo corresponde a una hectárea, la cual se dividió en 4 sub-parcelas de 0.25 hectáreas cada una. La toma de muestras se realizó en cada una, registrándose la información de esta en un día (diurna y nocturna) y completándose la información para la parcela (de una hectárea) en 4 días, con lo que se tiene por cada temporada un total de 6 parcelas muestreadas.

- La toma de muestras fue realizada por dos personas al mismo tiempo mediante caminatas aleatorias dentro de las unidades de muestreo (plotless units).

- Se empleó captura directa mediante la colecta manual, con la finalidad de registrar la araneofauna presente en hojarasca y sotobosque:

A) Ground hand collecting: este método permite la colecta hasta el nivel de la rodilla. Permite la captura de arañas que se encuentran en la hojarasca, suelo, leños y todo tipo de vegetación que no exceda el nivel de la rodilla.

B) Aerial hand collecting: consiste en la colecta desde el nivel de la rodilla hasta donde la mano del colector alcance (aprox. $2 \mathrm{~m}$ ). Este método permite la captura de arañas tejedoras y arañas libres en el follaje y/o en ramas de arbustos, hierbas altas, troncos y lianas (Sorensen et al., 2002).

- La toma de muestras se realizó durante un tiempo aproximado de 120 minutos $(60 \mathrm{~min} /$ pers.) de acuerdo a Sorensen et al. (2002). El primer día se realizaron evaluaciones preliminares para establecer la longitud del intervalo de tiempo a utilizar en el estudio, entre 60 y 90 minutos. El tiempo así definido se mantuvo en el total de unidades a evaluar.

- Siguiendo a Coddington et al. (1991) la colecta se realizó durante el día (7:00 AM - 9:00 AM) y durante la noche (7:00 PM - 9:00 PM) en las seis parcelas. Las colectas diarias (diurnas y nocturnas) y las colectas de ambos microhábitats se juntaron en una sola muestra, lo que dio cuatro muestras por parcela en un total de 24 muestras por época del año. Las muestras fueron colocadas en bolsas plásticas Whirl Pak de 4 oz en alcohol de $96^{\circ}$ llevando una etiqueta con un código asignado.

2. En laboratorio.

- Las muestras fueron analizadas en el laboratorio del Museo de Entomología "Klaus Raven Büller" de la Universidad Nacional Agraria La Molina y depositadas en la colección de arácnidos en viales de vidrio en alcohol de $75^{\circ}$.

- Los especímenes fueron determinados a nivel de especies y morfoespecies. Para cada especie se consideraron individuos adultos y juveniles sub adultos, para los cuales se aseguró su correspondencia. Para la identificación de los especímenes se utilizó las llaves del Dr. H. Levi.

\section{Análisis de los datos.}

Para el análisis de datos se utilizó el programa EstimateS versión 8.0 y se emplearon los siguientes índices de riqueza: Chao1, Jacknife de $1^{\circ}$ orden y Jacknife de $2^{\circ}$ orden que ayudan a estimar el número de especies que faltan por colectar basándose en la cuantificación de la rareza de especies colectadas (Toti et al. 2000 en Rico-G. et al., 2005), ACE basado en la abundancia que usan para cuantificar rareza, ICE basado en la incidencia: presencia y ausencia para cuantificar la rareza y Bootstrap que utiliza los datos de todas las especies colectadas para estimar la riqueza total, a través de la suma de la riqueza observada con el inverso de la proporción de muestras en que ocurre cada especie (Nogueira et al., 2006). Para el cálculo de los índices de Diversidad de Shannon (H') y Dominancia de Simpson (1-D) y el análisis de similitud de Bray-Curtis se utilizó el programa Past 1.56b. Para estimar la pendiente de la curva de Clench se utilizó el programa Statistica 
versión 6.0. Con la ecuación de Clench se puede determinar si el inventario es suficientemente fiable, a pesar de ser aún incompleto, cuando la pendiente es aproximadamente

$<0.1 \quad$ (Jimenez

Valverde \& Hortal, 2003), la cual ayuda a saber si el número de colectas realizadas es suficiente para registrar las especies existentes en un lugar.

\section{Resultados.}

En la época seca (ES) se recolectó un total de 1516 individuos, distribuidos en 25 géneros y 120 especies. En la época lluviosa (ELL) se

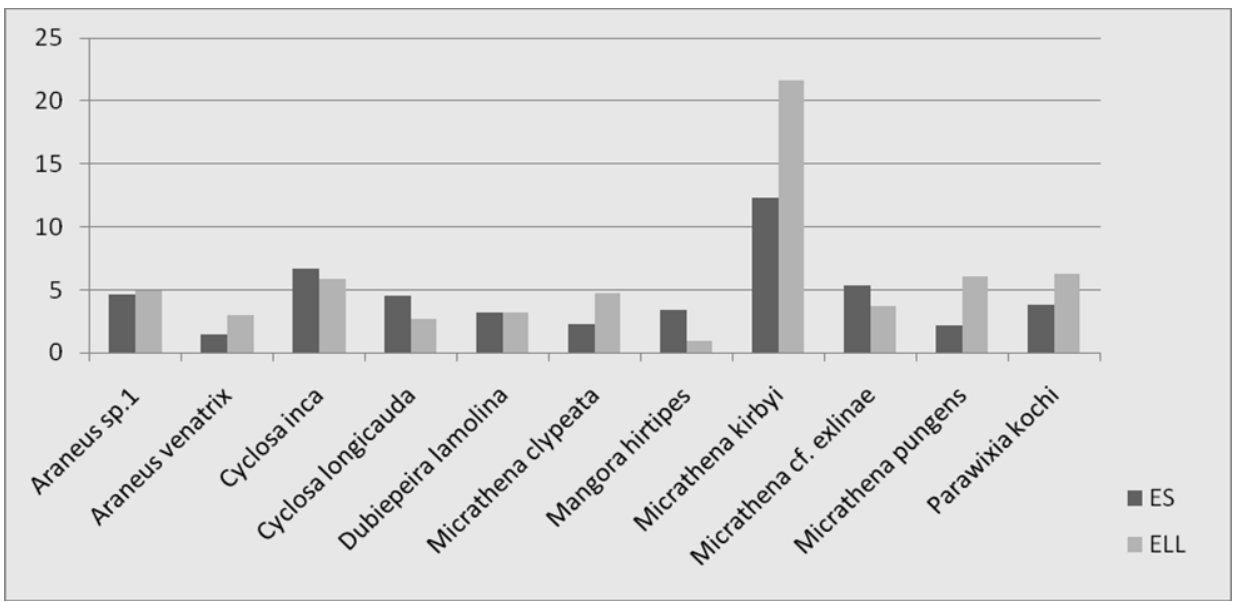

Figura 1. Porcentaje de la abundancia de las especies más comunes en época seca (ES) y época lluviosa (ELL). recolectó un total de 3173 individuos, en 24 géneros y 121 especies. Este alto número de individuos puede deberse a que la estación lluviosa concentra el pico de abundancia y riqueza de la araneofauna y es la época de reproducción de la mayoría de las especies, siendo un periodo en donde hay mayor oferta de alimento (Nogueira et al., 2006). Los géneros con mayor número de especies fueron Alpaida O.P.- Cambridge (ES: 12 spp., ELL: 15 spp.), Cyclosa Menge (ES: 6 spp., ELL: 8 spp.), Eustala Simon (ES: 10 spp., ELL: 13 spp.), Micrathena Sundevall (ES: 23 spp., ELL: 24 spp.), Parawixia F.O.P.- Cambridge (ES: 9 spp., ELL: 8 spp.) y Wagneriana F.O.P.- Cambridge (ES: 8 spp., ELL: 6 spp.) que, comparados con otros estudios en ecosistemas tropicales como los de Silva (1996) en el río Samiria, Silva \& Coddington (1996) en Pakitza, Nogueira (2006) en la Reserva de Morro Grande, Córdova \& Duarez (2001) en el Bajo Urubamba, se observa que estos géneros están entre los de mayor riqueza.

Las especies con mayor porcentaje de individuos colectados fueron Araneus sp.1 (4.6\% ES, 5\% ELL), Cyclosa longicauda (Taczanowski) (4.5\% ES, 2.7\% ELL), Cyclosa inca Levi (6.7\% ES, 6\% ELL), Dubiepeira lamolina Levi (3.2\% ES, 3.2\% ELL), Micrathena cf. exlinae (5.3\% ES, 3.7\% ELL), Micrathena clypeata (Walckenaer) (2,3\% ES, 4.7\% ELL), Micrathena kirbyi (Perty) (12\% ES, 22\% ELL) y Parawixia kochi (Taczanowski) (4 \% ES, 6.3\% ELL) (Figura 1). Los géneros con mayor número de individuos fueron Micrathena Sundevall (1814 ind.), Cyclosa Menge (539 ind.), Araneus Clerck (408 ind.), Parawixia F.O.P.- Cambridge (333 ind.) y Alpaida O.P.- Cambridge (300 ind.). En el Anexo 1 se puede observar las 154 especies y morfoespecies encontradas en los terrenos de CICRA. En el Anexo 2 se mencionan las 24 especies y morfoespecies que se recolectaron fuera de las parcelas en estudio y que no están presentes en la evaluación. 
encontraron 36 spp. con

un solo individuo (Singletons), 17 spp. con dos individuos (Doubletons). Mientras que en la época lluviosa (121 spp. colectadas) se encontraron 31 spp. Singleton y 14 spp. Doubleton. (Tabla 1). En ambas épocas el número de especies únicas es mayor que los Singletons, los Doubletons y especies duplicadas. Sólo en ELL la curva de Doubletons no parece disminuir tan progresivamente como las demás curvas (Figura 1, Figura 2). Es importante mencionar que el alto porcentaje de Singletons encontrados en los diferentes trabajos realizados, se debe a que los bosques tropicales son altamente diversos y que por lo tanto las especies dominantes son pocas y que predominan las especies raras (Silva, 1996; Florez-D., 1998; Benavides \& Florez, 2006; Silva \& Coddington, 1996), las cuales cumplen un papel importante en la generación de la mayoría de los estimadores que garantizan la confiabilidad de los resultados obtenidos, debido a que

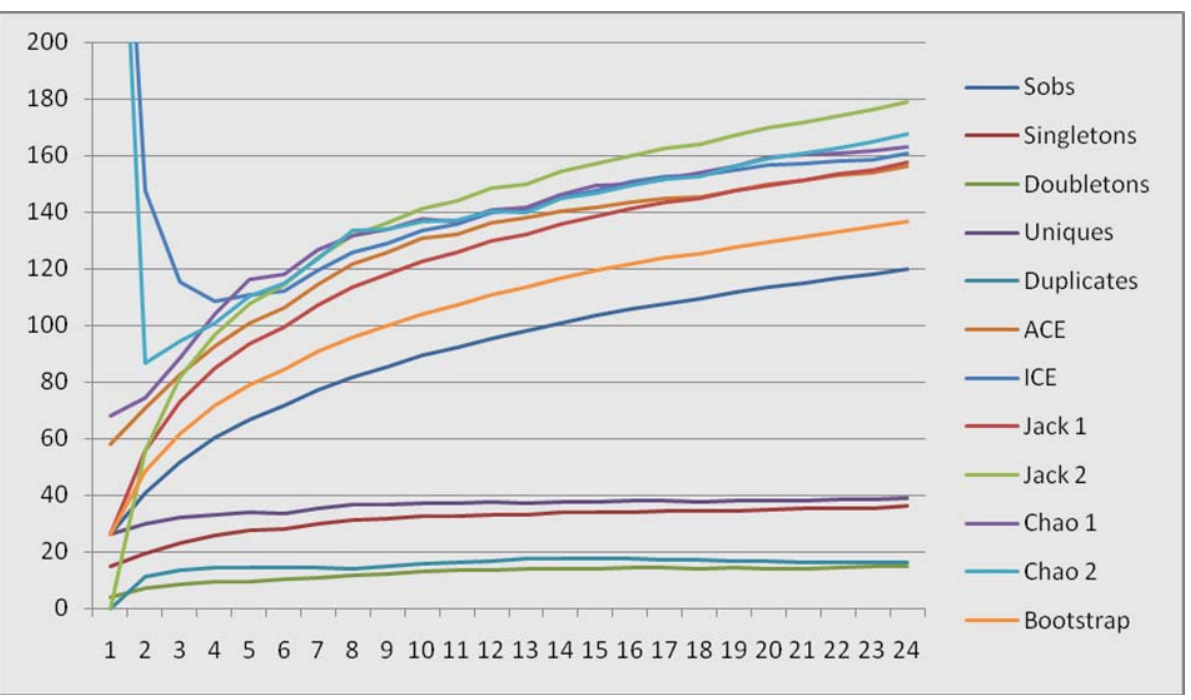

Figura 2. Riqueza de especies observadas y estimadas para la época seca (ES).

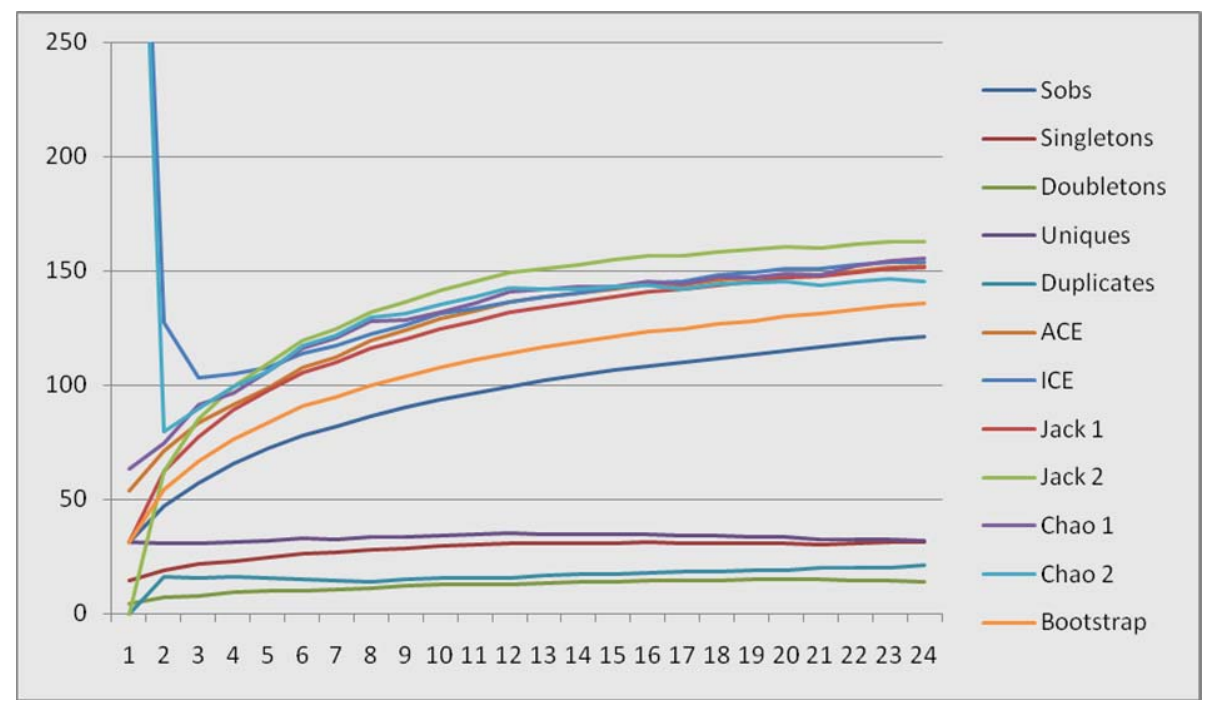

Figura 3. Riqueza de especies observadas y estimadas para la época lluviosa (ELL).

están basados en su cuantificación (Colwell, 2004 en Rico-G. et al., 2005).

Por otro lado el coeficiente de Bray-Curtis, muestra una similitud de especies entre ambas épocas del $52 \%$. Esta similitud - dada la enorme diferencia en el número de individuos - puede deberse a que la colecta de la ELL se realizó en un mes en el que las lluvias, a pesar de ser abundantes, ya habían disminuido en comparación con el mes anterior y las parcelas del estudio ya no se encontraban inundadas, por lo que la araneofauna de suelo probablemente ya se había restituido.

Se han realizado trabajos anteriores con arañas en Cusco y Madre de Dios (Silva \& Coddington, 1996; Silva, 1996; Córdova \& Duarez, 2001) obteniéndose una alta diversidad de especies, sobre todo en la familia Araneidae. En este estudio se obtuvo la mayor riqueza de esta familia registrada en la selva baja peruana, debido probablemente al tipo de muestreo realizado (colecta manual), tanto diurna como nocturna y en dos microhábitats diferentes, abarcando todo el bosque desde el suelo hasta los dos metros de altura.

Silva \& Coddington (1996) observaron en Pakitza (Madre de Dios) 145 morfoespecies de Araneidae en donde más del $50 \%$ de las especies colectadas estuvieron representadas por un solo individuo, Silva (1996) observó 207 especies de esta familia a lo largo del río Samiria (Loreto), en donde aproximadamente el $47 \%$ fueron Singletons, este alto número de especies fue producto de la utilización de cuatro métodos de colecta en seis microhábitats diferentes, los cuales cubrieron buena parte del bosque.

Según Nogueira et al. (2006), las colectas 
deberían hacerse de manera continua y no en varios periodos o por estaciones separadas, ya que el esfuerzo se puede estar diluyendo y la colecta volviéndose más superficial, lo que probablemente pudo haber sucedido en este caso, ya que sólo se hicieron dos evaluaciones de 24 días cada una. Esto explicaría el hecho de que las curvas de acumulación de especies muestren una clara ascensión, ya que en un ecosistema tan diverso como es la selva de Madre de Dios es necesario utilizar diferentes metodologías de muestreo, como las utilizadas por Silva (1996) y Silva \& Coddington (1996).

Tabla 1: Valores obtenidos en los análisis de riqueza de especies para cada época.

\begin{tabular}{llll}
\hline Riqueza observada & E. Seca & E. Lluviosa & Total \\
\hline Número de especies & 120 & 121 & 154 \\
Número de "Singletons" & 36 & 31 & \\
Número de doubletons & 15 & 14 & \\
Número de únicos & 39 & 32 & \\
Número de duplicados & 16 & 21 & \\
Adultos & 894 & 1896 & 2790 \\
\% del total de adultos & $32.04 \%$ & $67.95 \%$ & $100 \%$ \\
\hline Riqueza estimada & E. Seca & E. lluviosa & \\
\hline ACE & 156.25 & 151.76 & \\
ICE & 160.77 & 153.82 & \\
Chao1 & 157.38 & 151.67 & \\
Chao2 & 179.1 & 162.59 & \\
Jack1 & 163.2 & 155.32 & \\
Jack2 & 167.53 & 145.38 & \\
Bootstrap & 136.67 & 135.63 & \\
\hline
\end{tabular}

\section{Conclusiones.}

El total de individuos colectados fue de 4689 (2 790 adultos) en las dos épocas, de los cuales el $67.95 \%$ pertenecen a la ELL, pero fue en la ES en la que se encontró una mayor diversidad y riqueza.

En cuanto a especies raras se refiere, sólo el 29\% ES y el 26\% ELL tuvieron un solo individuo, debido tal vez a que sólo se utilizó una metodología de colecta, por lo tanto no se cubrieron todos los microhábitats en los que esta familia suele encontrarse.

En este estudio la mayoría de las curvas de los estimadores de riqueza y de especies raras no alcanzan una asíntota, mostrando de esta manera que es necesario un mayor esfuerzo de colecta.

Los resultados obtenidos muestran que la selva de Madre de Dios alberga una rica y abundante fauna de especies de Araneidae, incluyendo especies raras y algunas de ellas aún no descritas, siendo necesario hacer un examen más detallado del material recolectado. Cabe resaltar que a pesar de varios estudios realizados sobre la araneofauna en el Departamento de Madre de Dios, aún falta mucho por conocer, debido a que existen zonas como los Parques Nacionales Manu y Bahuaja - Sonene en las que las actividades humanas como tala indiscriminada de árboles, turismo, etc. son casi inexistentes.

El método de muestreo empleado en este estudio, condiciona los resultados obtenidos, ya que están sujetos a las observaciones y habilidad de colecta del investigador. Para obtener mejores resultados se sugiere a futuros estudios en CICRA, complementar la colecta manual con trampas pitfall, redadas, fogging entre otros.

\section{Agradecimientos}

A la Asociación para la Conservación de la Cuenca Amazónica (ACCA) por financiar los estudios de tesis de grado de la cual sale esta pequeña parte. A la profesora Clorinda Vergara, jefa del Museo de Entomología Klaus Raven Büller por brindarnos un espacio para poder trabajar nuestras arañas. A la Dra. Diana Silva del Museo de Historia Natural de la Universidad de San Marcos por su gran ayuda en la identificación de algunas especies. A nuestro amigo Eduardo Oyague por revisar el manuscrito y por su paciencia y buen humor para explicarnos como utilizar los programas estadísticos. A Laura Leibensperger de Harvard University - Museum of Comparative Zoology por brindarnos la bibliografía necesaria para la identificación de las especies. A todas aquellas personas anónimas que revisaron el manuscrito y nos brindaron sus sugerencias y finalmente a Carlos Deza por la traducción del resumen.

\section{Literatura citada.}

Benavides L. \& Florez E. 2006. Comunidades de arañas (Arachnida: Araneae) em microhábitats de dosel em bosques de tierra firme e igapó de La Amazônia colombiana. Revista Ibérica de Aracnología. 14: 49-62.

Candiani D.F., Indicatti R.P. \& Brescovit A.D. 2005. Composiçao e diversidade da araneofauna (Araneae) de serapilheira em tres florestas urbanas na cidade de São Paulo, São Paulo, Brasil. Biota Neotropica. 5 ( 1a).

Coddington J. \& Levi H. 1991. Systematics and evolution of spiders (Araneae). Ann. Rev. Ecol. Syst. 22: 565-592. Griswold Ch.E., Silva D., Peñaranda E. \& Larcher S.F. 1991. Desingning and Testing Sampling Protocols to Estimate Biodiversity in Tropical Ecosystems. Critical issues in Biodiversity Symposium.

Colwell R.K. 2004. EstimateS. Estatistical Estimation of Species Richness and Shared Species from Samples. Version 7. User's Guide and application at http://viceroy.edd.uconn.edu/estimates.

Córdova S. \& Duarez J. 2001. Diversity and Community Structure of the spiders of the Lower Urubamba Region, Perú. Pp. 67-71. In Alonso A., Dallmeier F. \& Campbell P. (Eds.). Urubamba: The diversity of Peruvian Rainforest. Smithsonian Institution.

Flórez-D. E. 1998. Estructura de comunidades de arañas (Araneae) en el Departamento Del Valle, Suroccidente de Colombia. Caldasia. 20 (2): 173-192.

Jiménez-Valverde A. \& Hortal J. 2003. Las Curvas de 
acumulación de especies y la necesidad de evaluar la calidad de los inventarios biológicos. Revista Ibérica de Aracnología. 8: 151-161.

Levi H.W. 1985. The spiny orb-weaver genera Micrathena and Chaetacis (Araneae: Araneidae). Bull. Mus. Comp. Zool. Harv. 150(8): 429-618.

1988. The Neotropical Orb-weaving spiders of the genus Alpaida (Araneae: Araneidae). Bull. Mus. Comp. Zool. Harv. 151(7): 365-487.

1991. The Neotropical and Mexican species of the orb - weaver genera Araneus, Dubiepeira and Aculepeira (Araneae: Araneidae). Bull. Mus. Comp. Zool. 152(4): 167-315.

. 1991. The Neotropical orb - weaver genera Edricus and Wagneriana (Araneae: Araneidae). Bull. Mus. Comp. Zool. 152(6): 363-415.

1992. Spiders of the Orb-weaver genus Parawixia in America (Araneae: Araneidae). Bull. Mus. Comp. Zool. Harv. 153(1): 1-46.

1992. American Neoscona and corrections to previous revisions of Neotropical orb - weavers (Araneae: Araneidae). Psyche. 99: 221-240.

1995. Orb-weaving spiders Actinosoma, Spilasma, Micrepeira, Pronous and four new genera (Araneae: Araneidae). Bull. Mus. Comp. Zool. Harv. 154(3): 153-213.

1995. The Neotropical Orb-weavers genera Metazygia (Araneae: Araneidae). Bull. Mus. Comp. Zool. Harv. 154(2): 63-151.

1996. The American orb-weavers Hypognatha, Encyosaccus, Xylethrus, Gasteracantha and Enacrosoma (Araneae: Araneidae). Bull. Mus. Comp. Zool. Harv. 155(3): 89-157.

2002. Keys to the genera of araneid orbweavers (Araneae, Araneidae) of the Americas. J. Arachnol. 30: 527-562.

2007. The orb weaver genus Mangora in South America (Araneae, Araneidae). Bull. Mus. Comp. Zool.
159(1): 1-144.

Nogueira A.A., Pinto-da-Rocha R. \& Brescovit A.D. 2006. Comunidade de aranhas orbitelas (Arachnida-Araneae) na regiao da Reserva Florestal do Morro Grande, Cotia, Sao Paulo, Brasil. Biota Neotropica. 6 (2).

Oliveira-Alves A., Lima Peres M.A., Alves M., da Silva G. \& Alardo L.R. 2005. Estudo das comunidades de aranhas (Arachnida: Araneae) em ambiente de Mata Atlantica no Parque Metropolitano de Pituacu - PMP, Salvador, Bahia. Biota Neotropica 5 (1a).

Pitman N.C.A. 2008. An overview of the Los Amigos watersheed, Madre de Dios, southeastern Peru. February 2007 version o fan unpublished report available from the autor at npitman@amazonconservation.org.

Platnick N.I. 2009. The world spider catalog, version 9.5. American Museum of Natural History, online at http://research.amnh.org/entomology/spiders/catalog/in dex.html. Acceso 22/VI/2009.

Rico-G. A., Beltrán J.P.A., Álvarez A.D. \& Florez E.D. 2005. Diversidad de Arañas (Arachnida: Araneae) en el Parque Nacional Natural Isla Gorgona, Pacífico Colombiano. Biota Neotropica. 5 (1a).

Silva D. 1996. Species composition and community structure of peruvian rainforest spiders: A case study from a seasonally inundated foresta long the Samiria river. Revue suisse de Zoologie, vol. hors série.: 597610.

\& Coddington J.A. 1996. Spiders of Pakitza (Madre de Dios, Perú): Species richness and Notes on Community Structure. Pp. 253-311. In Wilson D.E. \& Sandoval A. (Eds.). The Biodiversity of Southeastern Perú. Smithsonian Institution.

Sorensen L.L., Coddington J.A. \& Scharff N. 2002. Inventorying and estimating subcanopy spider diversity using semiquantitative sampling methods in an Afromontane forest. Environ. Entomol. 31(2): 319-330.

Anexo 1. Lista de las especies colectadas en época seca (ES) y época lluviosa (ELL) en Bajío (B) y Terraza (T) con el total de individuos.

\begin{tabular}{|c|c|c|c|c|c|c|}
\hline Especies & $\begin{array}{c}\text { ES } \\
\text { B }\end{array}$ & $\mathbf{T}$ & $\begin{array}{l}\text { BLL } \\
\text { B }\end{array}$ & $\mathbf{T}$ & Total & $\begin{array}{c}\% \\
\text { Total } \\
\end{array}$ \\
\hline Araneidae sp. 1 & 8 & & & 3 & 11 & 0.23 \\
\hline Araneidae sp. 2 & 4 & & & & 4 & 0.09 \\
\hline Araneidae sp. 3 & & 1 & & & 1 & 0.02 \\
\hline Araneidae sp. 4 & & & 1 & & 1 & 0.02 \\
\hline Araneidae sp. 5 & & & & 1 & 1 & 0.02 \\
\hline Araneidae sp. 6 & & & & 2 & 2 & 0.04 \\
\hline Araneidae sp. 7 & 2 & 60 & & & 62 & 1.32 \\
\hline Araneidae sp. 8 & 1 & & 2 & & 3 & 0.06 \\
\hline Araneidae sp. 9 & 1 & 1 & & & 2 & 0.04 \\
\hline Araneidae sp. 10 & & 1 & & 2 & 3 & 0.06 \\
\hline Araneidae sp. 11 & & 1 & & & 1 & 0.02 \\
\hline cf. Araneus & 3 & & 1 & & 4 & 0.09 \\
\hline cf. Eustala & 5 & 6 & 4 & 3 & 18 & 0.38 \\
\hline Acacesia benigna Glueck 1994 & & 16 & 5 & 38 & 59 & 1.26 \\
\hline Alpaida sp. 1 & 8 & 12 & & 9 & 29 & 0.62 \\
\hline
\end{tabular}


Anexo 1. Lista de las especies colectadas en época seca (ES) y época lluviosa (ELL) en Bajío (B) y Terraza (T) con el total de individuos.

\begin{tabular}{|c|c|c|c|c|c|c|}
\hline \multirow[b]{2}{*}{ Especies } & \multicolumn{2}{|l|}{ ES } & \multicolumn{2}{|l|}{ ELL } & \multirow[b]{2}{*}{ Total } & \multirow{2}{*}{$\begin{array}{c}\% \\
\text { Total } \\
\end{array}$} \\
\hline & B & $\mathbf{T}$ & B & $\mathbf{T}$ & & \\
\hline Alpaida sp. 2 & 1 & 2 & & & 3 & 0.06 \\
\hline Alpaida sp. 3 & 1 & 1 & & 4 & 6 & 0.13 \\
\hline Alpaida sp. 4 & 10 & 14 & 14 & 9 & 47 & 1.00 \\
\hline Alpaida sp. 5 & & 2 & 1 & 8 & 11 & 0.23 \\
\hline Alpaida sp. 6 & 1 & & & 1 & 2 & 0.04 \\
\hline Alpaida sp. 7 & & & 5 & 7 & 12 & 0.26 \\
\hline Alpaida sp. 8 & & & & 3 & 3 & 0.06 \\
\hline Alpaida sp. 9 & & & & 3 & 3 & 0.06 \\
\hline Alpaida sp. 10 & & 11 & & 3 & 14 & 0.30 \\
\hline Alpaida sp. 11 & & & & 1 & 1 & 0.02 \\
\hline Alpaida cf. Antonio & 2 & 1 & & 3 & 6 & 0.13 \\
\hline Alpaida cf. Dominica & & 3 & 2 & 8 & 13 & 0.28 \\
\hline Alpaida delicata (Keyserling 1892) & 13 & 9 & 27 & 34 & 83 & 1.77 \\
\hline Alpaida truncata (Keyserling 1865) & & 8 & 1 & 6 & 15 & 0.32 \\
\hline Alpaida vera Levi 1988 & & 5 & & 47 & 52 & 1.11 \\
\hline Amazonepeira herrera Levi 1989 & & 5 & 2 & 1 & 8 & 0.17 \\
\hline Araneus sp. 1 & 36 & 33 & 116 & 40 & 225 & 4.80 \\
\hline Araneus sp. 2 & & & & 1 & 1 & 0.02 \\
\hline Araneus guttatus (Keyserling 1865) & 9 & 23 & 18 & 12 & 62 & 1.32 \\
\hline Araneus tambopata Levi 1991 & & & & 2 & 2 & 0.04 \\
\hline Araneus venatrix (C.L. Koch 1838) & 11 & 11 & 23 & 73 & 118 & 2.52 \\
\hline Argiope argentata (Fabricius 1775) & 2 & 2 & & 1 & 5 & 0.11 \\
\hline Aspidolasius branicki (Taczanowski 1879) & 14 & 11 & 2 & 10 & 37 & 0.79 \\
\hline Bertrana sp. 1 & 1 & & & & 1 & 0.02 \\
\hline Bertrana elinguis (Keyserling 1883) & 8 & 4 & 14 & 5 & 31 & 0.66 \\
\hline Chaetacis cucharas Levi 1985 & 1 & & 5 & 2 & 8 & 0.17 \\
\hline Chaetacis necopinata (Chickering 1960) & 1 & & & 4 & 5 & 0.11 \\
\hline Chaetacis cornuta Taczanowski 1873 & 17 & & 28 & 5 & 50 & 1.07 \\
\hline Cyclosa sp. 1 & & 1 & & & 1 & 0.02 \\
\hline Cyclosa sp. 2 & & & 2 & & 2 & 0.04 \\
\hline Cyclosa sp. 3 & & & & 1 & 1 & 0.02 \\
\hline Cyclosa caroli (Hentz 1850) & & 1 & & 1 & 2 & 0.04 \\
\hline Cyclosa dianasilvae Levi 1999 & & & & 32 & 32 & 0.68 \\
\hline Cyclosa diversa (O.P.-Cambridge 1894) & 1 & & & 1 & 2 & 0.04 \\
\hline Cyclosa inca Levi 1999 & 43 & 59 & 72 & 115 & 289 & 6.16 \\
\hline Cyclosa longicauda (Taczanowski 1878) & 55 & 13 & 55 & 30 & 153 & 3.26 \\
\hline Cyclosa rubronigra Caporiacco 1947 & 23 & 6 & 19 & 9 & 57 & 1.22 \\
\hline Dubiepeira lamolina Levi 1991 & 23 & 26 & 60 & 43 & 152 & 3.24 \\
\hline Dubiepeira neptunina (Mello-Leitao 1948) & & & & 1 & 1 & 0.02 \\
\hline Enacrosoma anomalum (Taczanowski 1873) & 1 & & & & 1 & 0.02 \\
\hline Eriophora fuliginea (C.L. Koch 1838) & 4 & 4 & 4 & 12 & 24 & 0.51 \\
\hline Eustala sp. 1 & 1 & & 6 & & 7 & 0.15 \\
\hline Eustala sp. 2 & 1 & & & & 1 & 0.02 \\
\hline Eustala sp. 3 & & & 1 & 1 & 2 & 0.04 \\
\hline Eustala sp. 4 & & & 8 & 13 & 21 & 0.45 \\
\hline
\end{tabular}


Anexo 1. Lista de las especies colectadas en época seca (ES) y época lluviosa (ELL) en Bajío (B) y Terraza (T) con el total de individuos.

\begin{tabular}{|c|c|c|c|c|c|c|}
\hline Especies & $\begin{array}{c}\text { ES } \\
\text { B } \\
\end{array}$ & $\mathbf{T}$ & ELL & $\mathbf{T}$ & Total & $\begin{array}{c}\% \\
\text { Total } \\
\end{array}$ \\
\hline Eustala sp. 5 & & & 2 & 34 & 36 & 0.77 \\
\hline Eustala sp. 6 & & 1 & 2 & 21 & 24 & 0.51 \\
\hline Eustala sp. 7 & & & 1 & 8 & 9 & 0.19 \\
\hline Eustala sp. 8 & & 2 & & 3 & 5 & 0.11 \\
\hline Eustala sp. 9 & & 1 & & & 1 & 0.02 \\
\hline Eustala sp. 10 & & & & 1 & 1 & 0.02 \\
\hline Eustala sp. 11 & & & & 1 & 1 & 0.02 \\
\hline Eustala sp. 12 & 13 & 6 & 1 & & 20 & 0.43 \\
\hline Eustala sp. 13 & 1 & 1 & & & 2 & 0.04 \\
\hline Eustala sp. 14 & & & & 1 & 1 & 0.02 \\
\hline Eustala sp. 15 & & 21 & & 56 & 77 & 1.64 \\
\hline Eustala sp. 16 & 3 & 5 & 2 & 1 & 11 & 0.23 \\
\hline Eustala sp. 17 & & 1 & & & 1 & 0.02 \\
\hline Hypognatha sp. 1 & & 1 & & & 1 & 0.02 \\
\hline Hypognatha sp. 2 & & 2 & & 1 & 3 & 0.06 \\
\hline Hypognatha sp. 3 & & 1 & & & 1 & 0.02 \\
\hline Hypognatha sp. 4 & & 1 & 1 & & 2 & 0.04 \\
\hline Hypognatha sp. 5 & & & & 3 & 3 & 0.06 \\
\hline Hypognatha matisia Levi 1996 & & 2 & 1 & & 3 & 0.06 \\
\hline Hypognatha nasuta & 6 & & 21 & 7 & 34 & 0.73 \\
\hline Hypognatha navio Levi 1996 & 1 & & & 1 & 2 & 0.04 \\
\hline Mangora sp. 1 & 5 & 21 & & 31 & 57 & 1.22 \\
\hline Mangora amacayacu Levi 2007 & 1 & & 1 & 2 & 4 & 0.09 \\
\hline Mangora dianasilvae Levi 2007 & 4 & 3 & 26 & 3 & 36 & 0.77 \\
\hline Mangora hirtipes (Taczanowski 1878) & 6 & 46 & & 30 & 82 & 1.75 \\
\hline Mangora tambo Levi 2007 & 3 & 6 & 18 & 12 & 39 & 0.83 \\
\hline Manogea porracea (C. L. Koch 1838) & 7 & 7 & 9 & 13 & 36 & 0.77 \\
\hline Metazygia sp. 1 & & & & 7 & 7 & 0.15 \\
\hline Metazygia sp. 2 & & 2 & & 2 & 4 & 0.09 \\
\hline Metazygia castaneoscutata (Simon 1895) & & 2 & & 30 & 32 & 0.68 \\
\hline Metazygia tapa Levi 1995 & & 1 & 2 & 3 & 6 & 0.13 \\
\hline Micrathena sp. 1 & & 1 & 1 & 1 & 3 & 0.06 \\
\hline Micrathena sp. 2 & & & 1 & & 1 & 0.02 \\
\hline Micrathena sp. 3 & & & & 6 & 6 & 0.13 \\
\hline Micrathena sp. 4 & & & & 1 & 1 & 0.02 \\
\hline Micrathena sp. 5 & 2 & 3 & 1 & & 6 & 0.13 \\
\hline Micrathena sp. 6 & 1 & 2 & & & 3 & 0.06 \\
\hline Micrathena sp. 7 & & 3 & & & 3 & 0.06 \\
\hline Micrathena sp. 8 & & 2 & & & 2 & 0.04 \\
\hline Micrathena sp. 9 & & & 1 & & 1 & 0.02 \\
\hline Micrathena sp. 10 & & & & 1 & 1 & 0.02 \\
\hline Micrathena acuta (Walckenaer 1842) & & 5 & 3 & 8 & 16 & 0.34 \\
\hline Micrathena coca Lev, 1985 & 1 & & & & 1 & 0.02 \\
\hline
\end{tabular}


Anexo 1. Lista de las especies colectadas en época seca (ES) y época lluviosa (ELL) en Bajío (B) y Terraza (T) con el total de individuos.

\begin{tabular}{|c|c|c|c|c|c|c|}
\hline \multirow[b]{2}{*}{ Especies } & \multicolumn{2}{|l|}{ ES } & \multicolumn{2}{|l|}{ ELL } & \multirow[b]{2}{*}{ Total } & \multirow{2}{*}{$\begin{array}{c}\% \\
\text { Total } \\
\end{array}$} \\
\hline & B & $\mathbf{T}$ & B & $\mathbf{T}$ & & \\
\hline Micrathena clypeata (Walckenaer 1805) & 4 & 31 & 30 & 120 & 185 & 3.95 \\
\hline Micrathena embira Levi 1985 & & & & 1 & 1 & 0.02 \\
\hline Micrathena evansi Chickering 1960 & & & & 5 & 5 & 0.11 \\
\hline Micrathena exlinae Levi 1985 & & 2 & 2 & 1 & 5 & 0.11 \\
\hline Micrathena flaveola (Perty 1839) & 3 & & & 1 & 4 & 0.09 \\
\hline Micrathena furcata (Hahn 1822) & 19 & 4 & 66 & 8 & 97 & 2.07 \\
\hline Micrathena kirbyi (Perty 1833) & 31 & 156 & 76 & 608 & 871 & 18.58 \\
\hline Micrathena lata Chickering 1960 & 4 & 1 & 2 & 4 & 11 & 0.23 \\
\hline Micrathena macfarlanei Chickering 1961 & 1 & & & 3 & 4 & 0.09 \\
\hline Micrathenamiles Simon 1895 & 15 & 26 & 9 & 30 & 80 & 1.71 \\
\hline Micrathena pungens (Walckenaer 1842) & 11 & 22 & 50 & 143 & 226 & 4.82 \\
\hline Micrathena saccata (C. L. Koch 1836) & 1 & & & & 1 & 0.02 \\
\hline Micrathena schreibersi (Perty 1833) & 7 & 1 & 8 & & 16 & 0.34 \\
\hline Micrathena triangularis (C. L. Koch 1836) & & 1 & & 7 & 8 & 0.17 \\
\hline Micrathena triangularispinosa (De Geer 1778) & 1 & 1 & & & 2 & 0.04 \\
\hline Micrathena vigorsi (Pert, 1833) & 9 & & 4 & 2 & 15 & 0.32 \\
\hline Micrathena ca. exlinae & 76 & 5 & 113 & 5 & 199 & 4.24 \\
\hline Micrathena ca. triangularispinosa & 3 & 5 & 12 & 20 & 40 & 0.85 \\
\hline Micrepeira fowleri Levi 1995 & & 1 & & 2 & 3 & 0.06 \\
\hline Micrepeira pachitea Lev, 1995 & & 19 & & & 19 & 0.41 \\
\hline Ocrepeira camaca Levi 1993 & 1 & 1 & 1 & & 3 & 0.06 \\
\hline Ocrepeira covillei Levi 1993 & 2 & 9 & 7 & 18 & 36 & 0.77 \\
\hline Parawixia sp. 1 & 1 & & & 1 & 2 & 0.04 \\
\hline Parawixia sp. 2 & 2 & 7 & & & 9 & 0.19 \\
\hline Parawixia sp. 3 & 2 & 2 & & & 4 & 0.09 \\
\hline Parawixia sp. 4 & 3 & 2 & & & 5 & 0.11 \\
\hline Parawixia sp. 5 & & 5 & & 4 & 9 & 0.19 \\
\hline Parawixia sp. 6 & & 5 & & 4 & 9 & 0.19 \\
\hline Parawixia sp. 7 & & & 1 & 1 & 2 & 0.04 \\
\hline Parawixia sp. 8 & & & 1 & & 1 & 0.02 \\
\hline Parawixia sp. 9 & & & & 2 & 2 & 0.04 \\
\hline Parawixia divisoria Levi 1992 & & 1 & & & 1 & 0.02 \\
\hline Parawixia hypocrita (O. P.-Cambridge 1889) & 2 & 4 & 14 & 11 & 31 & 0.66 \\
\hline Parawixia kochi (Taczanowski 1873) & 27 & 30 & 113 & 88 & 258 & 5.50 \\
\hline Spilasma duodecimguttata (Keyserling 1879) & & & 1 & 3 & 4 & 0.09 \\
\hline Spintharidius rhomboidalis Simon 1893 & 2 & 9 & & & 11 & 0.23 \\
\hline Testudinaria sp. 1 & & & & 2 & 2 & 0.04 \\
\hline Verrucosa sp. 1 & 2 & & & & 2 & 0.04 \\
\hline Verrucosa sp. 2 & & 1 & & 1 & 2 & 0.04 \\
\hline Verrucosa sp. 3 & & 4 & & & 4 & 0.09 \\
\hline Verrucosa sp. 4 & & 1 & & & 1 & 0.02 \\
\hline Verrucosa sp. 5 & & & 1 & 1 & 2 & 0.04 \\
\hline Wagneriana hassleri Levi 1991 & & 4 & 9 & 9 & 22 & 0.47 \\
\hline Wagneriana lechuza Levi 1991 & 1 & 3 & 2 & 9 & 15 & 0.32 \\
\hline Wagneriana neblina Levi 1991 & & 1 & & & 1 & 0.02 \\
\hline
\end{tabular}


Anexo 1. Lista de las especies colectadas en época seca (ES) y época lluviosa (ELL) en Bajío (B) y Terraza (T) con el total de individuos.

\begin{tabular}{lrrrrrrr}
\hline & ES & \multicolumn{2}{c}{ ELL } & & & \% \\
Especies & & & & & Total & Total \\
\hline Wagneriana pakitza Levi 1991 & B & \multicolumn{1}{c}{ T } & B & T & Tot \\
Wagneriana roraima Levi 1991 & & 2 & & 1 & 3 & 0.06 \\
Wagneriana silvae Levi 1991 & 5 & 6 & & 2 & 13 & 0.28 \\
Wagneriana taim Levi 1991 & 9 & 25 & 11 & 28 & 73 & 1.56 \\
Wagneriana tayos Levi 1991 & 1 & & & & 1 & 0.02 \\
Xylethrus scrupeus Simon 1895 & & 5 & 2 & 3 & 10 & 0.21 \\
& & 1 & & & 1 & 0.02 \\
\hline
\end{tabular}

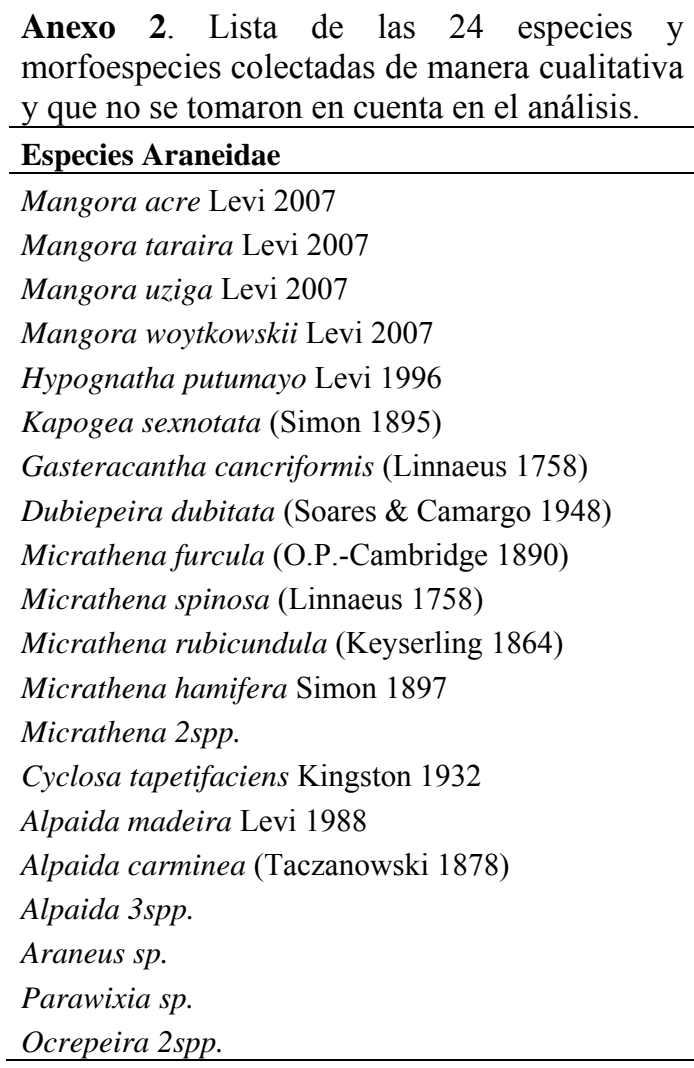

1 Museo de Entomología “Klaus Raven Büller”. Universidad Nacional Agraria La Molina. Av. La Molina s/n. La Molina, Lima - Perú, majoarchi79@hotmail.com.

2 Museo de Entomología "Klaus Raven Büller”. Universidad Nacional Agraria La Molina. Av. La Molina s/n. La Molina, Lima - Perú, juanmanuel_a_n@yahoo.es. 